\title{
Editorial
}

\section{What a 'Ku'incidence!: parallel discoveries of a new DNA repair factor}

\author{
M Mistrik ${ }^{\star, 1}$ and J Bartek $k^{*, 1,2}$ \\ Cell Death and Differentiation (2015) 22, 888-889; doi:10.1038/cdd.2015.32
}

\begin{abstract}
Maintenance of genomic integrity is a prerequisite for life, as we know it, and a fundamental biological process that relies on a carefully orchestrated cellular network of DNA damage sensors, signal transducers and diverse effectors including multiple DNA repair pathways. ${ }^{1,2}$ The significance of such DNA damage response (DDR) machinery is perhaps best documented by the severe organismal consequences of defects in many of the DDR components, ranging from developmental disorders, life-threatening immunodeficiency and neurodegenerative syndromes to cancer and premature aging. ${ }^{1,2}$ To improve diagnosis and treatment, and possibly implement even preventive measures to limit such grave pathologies, it is crucial to better understand the DDR network and identify the key components of the DNA damage checkpoints, chromatin remodeling and DNA repair pathways. One such important new component of a major pathway that repairs DNA double strand breaks (DSBs, arguably the most toxic type of DNA lesions) has just been identified by Craxton et al. $^{3}$ as reported elsewhere in this issue of Cell Death and Differentiation.
\end{abstract}

The DNA DSBs can be caused by multiple genotoxic insults including ionizing radiation (IR), a range of chemicals and drugs, as well as endogenous causes such as collapse of replication forks under conditions of DNA replication stress, for example. ${ }^{1,2}$ Consistent with the grave threat from unrepaired or misrepaired DSBs, all eukaryotes evolved mechanisms to repair these lesions, the two major pathways being homologous recombination repair and non-homologous end joining (NHEJ). ${ }^{1,2}$ It is the latter, NHEJ pathway, ${ }^{4,5}$ in which the new DNA repair protein named XLS (XRCC4-Like-Small protein; so far known only as c9orf142) by Craxton et al. ${ }^{3}$ has an important role. NHEJ repair requires a set of so-called core factors including Ku70 (also known as XRCC6), Ku80 (also known as XRCC5), DNA-PKcs, XLF and the XRCC4/DNA ligase IV complex. ${ }^{4,5}$ In addition, when more complex 'dirty' broken ends of DNA need re-joining, also several accessory NHEJ factors join the core proteins and participate in such more demanding DSB repair process (Figure 1). The work of Craxton et al. ${ }^{3}$ now extends the list of the workhorse core factors of NHEJ by adding XLS to the list.

So how did the authors discover XLS? To search for potential novel NHEJ factors, Craxton et al. generated a human cell

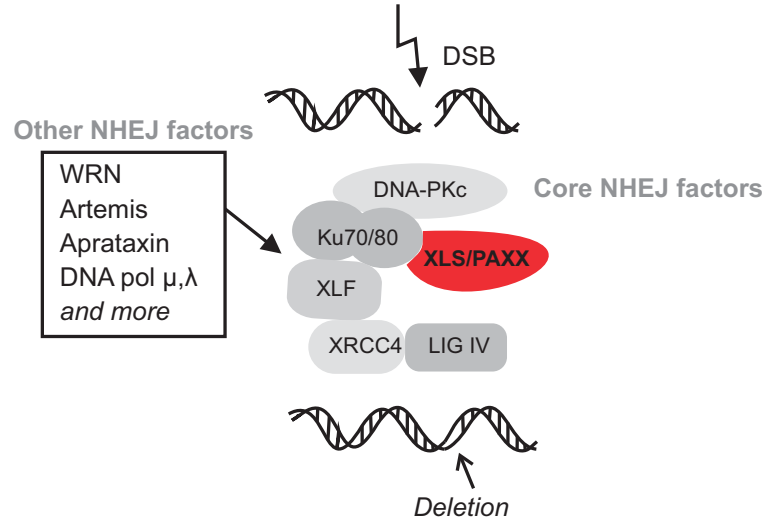

Figure $1 \mathrm{XLS} / \mathrm{PAXX}$ as a new component of the non-homologous end joining (NHEJ) DNA repair pathway. NHEJ is the main DNA double strand break (DSB) repair mechanism in G1 and early S phase. Depending on the nature of the DSB ('clean' versus 'dirty' ends) the NHEJ process involves multiple factors and preprocessing steps leading to deletions of various extents. The newly identified XLS/ PAXX protein qualifies as a core NHEJ factor (see main text for details)

line expressing tagged version of the DNA-PKcs, coimmunoprecipitated endogenous cellular proteins associated with the tagged DNA-PKcs, and used mass spectrometry to reveal a previously uncharacterized component of the NHEJ complex, so far known simply as c9orf142. Using computerbased modeling, the authors went on to predict the structure for this new NHEJ component to be very similar to XRCC4, which inspired the name XLS. ${ }^{3}$ Consistent with XLS playing a role in NHEJ repair, the authors could demonstrate that XLS interacted also with the other known core NHEJ proteins and, when endogenous XLS was depleted from cells, this impaired repair of DSBs. ${ }^{3}$

As is often the case in the current globally highly competitive research climate, this new NHEJ factor was also identified by other groups in parallel (in two other laboratories), through different strategies, and leading to very similar, mutually complementary conclusions. The first of the three reports on this new NHEJ factor was published by Ochi et al. ${ }^{6}$ who found c9orf142 as a NHEJ protein through application of bioinformatics and structure-guided approaches. This group obtained

${ }^{1}$ Institute of Molecular and Translational Medicine, Faculty of Medicine and Dentistry, Palacky University, Olomouc, Czech Republic and ${ }^{2}$ Danish Cancer Society Research Center, Copenhagen, Denmark

${ }^{*}$ Corresponding author: M Mistrik or Jiri Bartek, Danish Cancer Society, Strandboulevarden 49, Copenhagen DK-2100, Denmark. Tel: +4535257357; Fax: +4535271811; E-mail: martin.mistrik@upol.cz or jb@cancer.dk 
a crystal structure of c9orf142 and named this factor PAXX (PAralog of XRCC4) and XLF. They further showed a direct interaction of PAXX with $\mathrm{Ku}$, dynamic recruitment of PAXX to DSB lesions (using the laser microirradiation approach, commonly used to study the interactions of DDR proteins with the DSB ends or DSB-flanking chromatin ${ }^{7}$ ), and the role of PAXX in NHEJ in response to IR. ${ }^{6}$ In mechanistic terms, Ochi et al. documented the ability of c9orf142/PAXX to promote the assembly of the NHEJ core complex under DNA damage conditions, and to enhance the Ku-dependent DNA ligation step. ${ }^{6}$

The third report on the discovery and characterization of this new NHEJ protein, by Xing et al., ${ }^{8}$ also coined the name PAXX for the c9orf142 factor, and also documented its direct binding to Ku and the crystal structure reminiscent of those for XRCC4 and XLF. The authors showed that PAXX-depleted cells were more sensitive to DSB-causing genotoxic insults, and could distinguish two modes of PAXX engagement in the NHEJ process, dependent on the degree of complexity of the DNA ends flanking the DSBs. Thus, in NHEJ repair of complex 'dirty' DSBs (caused by IR), PAXX cooperated with XLF, while in response to simple - 'clean' DSBs (in response to a topoisomerase inhibitor treatment) the functions of PAXX and XLF were mutually redundant. ${ }^{8}$

Overall, the data sets presented in these three studies ${ }^{3,6,8}$ establish PAXX/XLS as a new component of the NHEJ repair machinery, a process critical for the maintenance of genomic integrity. Although important, these findings represent just the first step toward understanding the biological role of PAXX/ XLS, and raise a host of burning questions about its significance for biomedicine. For example, as physiological DSBs occur during the functional maturation of lymphocytes and NHEJ repair is required for proper rearrangement of the immunoglobulin genes, it would be interesting to examine whether knockout of the $P A X X / X L S$ gene would cause some form of immunodeficiency, a scenario seen in animal genetic models and human patients with hereditary defects in some other DNA repair factors. ${ }^{1,2,4,5,9,10}$ In this context, it is intriguing that unlike the phyllogenetically highly conserved XRCC4 and $\mathrm{XLF}, \mathrm{PAXX} / \mathrm{XLS}$ is not present in yeast and most invertebrates. Such limited evolutionary expression is reminiscent of DNA-PKcs, suggesting co-evolution of these two factors, possibly to cope with the complex DSB ends encountered during the $\mathrm{V}(\mathrm{D}) \mathrm{J}$ recombination of vertebrate immunoglobulin genes in B-cells, ${ }^{8,9}$ thereby contributing to robust immune response and defense against environmental pathogens.

Another area of anticipated lively research on PAXX/XLS's potential involvement in tumorigenesis, a complex evolutionary process in which activated DDR checkpoints may serve as an intrinsic barrier against tumor progression ${ }^{11,12}$ and where alterations of various DNA repair pathways in cancer cells contribute to the often stunning degree of genetic heterogeneity. ${ }^{13}$ For example, is the gene encoding PAXX/XLS-mutated or otherwise altered in any type of cancer? As NHEJ repair seems involved in cancerassociated chromosomal translocations, ${ }^{9,13}$ could aberrant activity of PAXX/XLS contribute to formation of the powerful oncogenic events that target the immunoglobulin gene loci in lymphomas, and possibly fuel formation of some fusion oncogenes in solid cancers?

Last but not least, given the emerging role of PAXX/XLS in NHEJ-mediated DNA repair, and the recent boom of innovative cancer treatments, including drugs such as PARP inhibitors that target defects in DSB repair pathways, ${ }^{14,15}$ it would be worthwhile to assess any value of PAXX/XLS status as a potential biomarker of treatment responses to both standard-of-care genotoxic treatments such as radiotherapy or chemotherapy, and any relevant upcoming DDR-targeting therapies. Indeed, as exemplified by responses to PARP inhibitors, functional shifts in the balance between the HR and NHEJ pathways have a major impact on the outcome of such treatment, and the status of several DSB-response factors promise to provide useful biomarkers ${ }^{16-20}$ to guide selection of subsets of patients for such targeted treatments in the future.

\section{Conflict of Interest}

The authors declare no conflict of interest.

Acknowledgements. We thank the Czech National Program of Sustainability (LO1304), the Kellner Family Foundation and the Danish Cancer Society for support.

\footnotetext{
1. Jackson SP, Bartek J. Nature 2009; 461: 1071-1078.

2. Ciccia A, Elledge SJ. Cell 2010; 40: 179-204.

3. Craxton A et al. Cell Death Differ 2015 in press.

4. Grundy GJ et al. DNA Repair (Amst) 2014; 17: 30-38.

5. Waters CA et al. DNA Repair (Amst) 2014; 17: 39-51.

6. Ochi T et al. Science 347: 185-188.

7. Bekker-Jensen S et al. J Cell Biol 2006; 173: 195-206.

8. Xing $\mathrm{M}$ et al. Nat Comm 2015; 6: 6233.

9. Kumar V, Alt FW, Oksenych V. DNA Repair 2014; 16: 11-22.

10. Devgan SS et al. Cell Death Differ 2011; 18: 1500-1506.

11. Halazonetis TD, Gorgoulis VG, Bartek J. Science 2008; 319: 1352-1355.

12. Evangelou K et al. Cell Death Differ 2013; 20: 1485-1497.

13. Burrell RA et al. Nature 2013; 501: 338-345.

14. Lord CJ, Tutt AN, Ashworth A. Annu Rev Med 2015; 66: 455-470.

15. Bouwman P, Jonkers J. Clin Cancer Res 2014; 20: 540-547.

16. Bouwman $P$ et al. Nat Struct Mol Biol 2010; 17: 688-695.

17. Oplustilova L et al. Cell Cycle 2012; 11: 3837-3850.

18. Di Virgilio $M$ et al. Science 2013; 339: 711-715.

19. Watanabe $\mathrm{S}$ et al. Nat Struct Mol Biol 2013; 20: 1425-1433.

20. Xu G et al. Nature 2015 (in press)
} 